\title{
Match Success Trends in United States Ophthalmology Residency Programs
}

\author{
Todd H. Driver, MD ${ }^{1} \quad$ Allison R. Loh, MD ${ }^{2}$ Fei Yu, $\mathrm{PhD}^{1} \quad$ Steven D. Pletcher, MD ${ }^{3}$ Ayman Naseri, MD ${ }^{4,5}$
}

1 Stein-Doheny Eye Institute, Department of Ophthalmology, University of California Los Angeles, Los Angeles, California

2 Department of Ophthalmology, Casey Eye Institute, Oregon Health and Science University, Portland, Oregon

${ }^{3}$ Department of Otolaryngology-Head and Neck Surgery, University

of California San Francisco, San Francisco, California

${ }^{4}$ Department of Ophthalmology, University of California San

Francisco, San Francisco, California

${ }^{5}$ Department of Ophthalmology, San Francisco Veterans Affairs

Medical Center, San Francisco, California

J Clin Acad Ophthalmol 2016;8:e42-e45.
Address for correspondence Ayman Naseri, MD, Department of Ophthalmology, University of California San Francisco, 10 Koret Way, San Francisco, CA 94121 (e-mail: Ayman.Naseri@va.gov).

\author{
Abstract \\ Keywords \\ - ophthalmology \\ residency \\ - match rate \\ - medical education
}

Background Medical students in the United States are inconsistently exposed to ophthalmology. Not only is this eroding U.S. medical graduates' ability to diagnose and treat ophthalmologic conditions, it may potentially affect interest in the specialty.

Methods To assess medical student's interest in pursuing ophthalmology training, this study sought to evaluate the match rate trend among U.S. medical school seniors applying into a U.S. ophthalmology residency and compare the trend to another traditionally competitive surgical subspecialty, otolaryngology (ENT).

Results From 2009 to 2013, the rate of successful residency matching for ophthalmology increased ( $12 \%$ increase per year in the odds of matching [ $95 \% \mathrm{Cl}: 1.04,1.20]$ ), while the ENT match rate decreased ( $7 \%$ decrease per year in the odds of matching (95\% Cl: 0.87-0.99). The ophthalmology match rate increased despite the fact that the expansion in the number of graduating U.S. medical students outpaced the increasing number of ophthalmology and ENT residency positions.

Conclusion The increasing match rate in ophthalmology is surprising and suggests that U.S. medical student's interest in ophthalmology may be waning. Future investigations are needed to determine the reasons behind the increasing match rate and the potential decreasing interest of medical students toward ophthalmology.
A decreasing proportion of graduating U.S. seniors are choosing primary care specialties, such as internal medicine, pediatrics, and family medicine. ${ }^{1}$ With this decrease, medical student's preference has shifted to residencies with controllable lifestyle, income, and shorter duration of postgraduate training. $^{2,3}$ This has been reflected in the increase in popularity of certain specialties thought to have controllable lifestyles such as anesthesiology, radiology, plastic surgery, orthopedic surgery, and emergency medicine. ${ }^{1}$ A previous study categorized ophthalmology as a "controllable lifestyle"

received

October 19, 2015

accepted after revision

August 29, 2016
DOI http://dx.doi.org/

$10.1055 / \mathrm{s}-0036-1593729$ ISSN 2379-0539. specialty, but none have characterized its desirability among graduating U.S. seniors over time.

There are reasons to believe that ophthalmology may not be as sought after as it has been anecdotally believed to be. Medical students in the United States are no longer being consistently exposed to ophthalmology. ${ }^{4}$ Without preliminary exposure to the field, medical students do not have the opportunity to evaluate the profession and select it as a career path. While ophthalmology and other surgical subspecialties have traditionally been characterized as among the most desirable and competitive specialties,
Copyright $\odot 2016$ by Thieme Medical Publishers, Inc., 333 Seventh Avenue, New York, NY 10001, USA. Tel: +1(212) 584-4662.
License terms

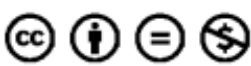


no studies have recently investigated longitudinal medical student's preferences toward ophthalmology.

Given the lack of clarity about the desirability of ophthalmology among graduating U.S. medical students, we sought to investigate the following question: Is medical students' interest in ophthalmology changing? To answer this question, we evaluated the longitudinal trend of match rates of U.S. medical students applying to ophthalmology. We also evaluated the match rates for another traditionally competitive surgical subspecialty, otolaryngology (ENT). for comparison.

\section{Methods}

We obtained de-identified and redacted match data from the San Francisco Matching Program (SF Match), ${ }^{5}$ the National Resident Matching Program (NRMP), ${ }^{6}$ and the Association of American Medical Colleges (AAMC) ${ }^{7}$ from 2009 to 2013. The total number of graduating U.S. medical students was also collected from the AAMC database. ${ }^{7}$ Institutional board exemption was obtained from the University of California, San Francisco Committee on Human Research. An author (T.H. D.) calculated the match rate trends from 2009 to 2013 among graduating U.S. medical school seniors (U.S. seniors). The match rate was defined as the total number of U.S. seniors who successfully matched in either ophthalmology or ENT residency training program divided by the total number of U.S. seniors who registered for the respective matches.

A Cochran-Armitage Trend Test was conducted to calculate the linear trend in match rate for both ophthalmology and ENT from 2009 to 2013. Additionally, an odds ratio was calculated to evaluate the odds of matching in each specialty from year to year. Finally, Fisher exact test was used to calculate the difference in overall match rate between ENT and ophthalmology from 2009 to 2013. Statistical analyses were performed using SAS 9.4 (SAS Institute, Inc., Cary, NC).

\section{Results}

The data show that from the years 2009 to 2013, ENT and ophthalmology had diverging match rates $(p<0.05)$ ( - Fig. 1 ). When analyzing the odds in matching from year 2009 to 2013,

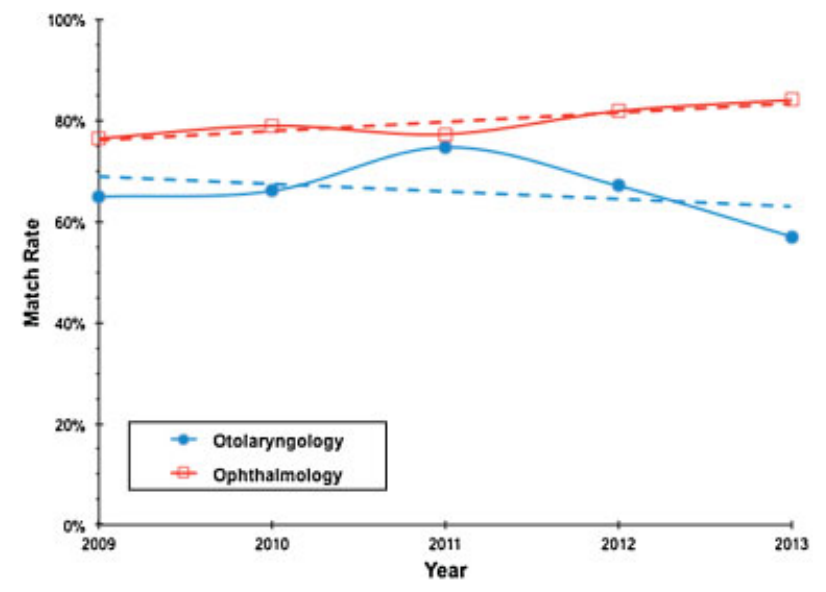

Fig. 1 Trend in ophthalmology versus ENT match rate from 2009 to 2013.
ENT showed an odds ratio of 0.93 (95\% CI: 0.87-0.99), corresponding with a $7 \%$ decrease per year in the odds of matching from 2009 to 2013. Conversely, when analyzing the odds of matching in ophthalmology over the same time period, the odds ratio was 1.12 (95\% confidence interval [CI]: 1.04, 1.20), corresponding to a $12 \%$ increase per year in the odds of matching from 2009 to 2013. When comparing the available residency spots in ENT and ophthalmology between 2009 and 2013, ENT experienced a $6 \%$ increase in residency spots, while ophthalmology had a $0.2 \%$ increase in residency spots. When comparing the number of graduating U.S. medical students from 2009 and 2013, there was an approximate $11.6 \%$ increase in graduating U.S. senior medical students ( - Table 1). Additionally, the comparison in the overall match rate between ENT and ophthalmology from 2009 to 2013 shows that ENT had a significantly lower match rate compared with ophthalmology $(p<0.0001)$.

\section{Discussion}

Overall, this study found that successful matching into ophthalmology for graduating U.S. seniors has become less competitive over time, as the odds of matching into ophthalmology have been increasing since the year 2009. In contrast, the odds of matching into ENT, a similar surgical subspecialty, has decreased over the same time period.

There may be several reasons for the increasing odds of matching into ophthalmology. Previous studies have found that factors such as controllable lifestyle, years of graduate medical education, and perceived income affect medical student's career preference, thereby suggesting that ophthalmology is not perceived to be able to fulfill these criteria as strongly as it was previously. ${ }^{2,8}$ Additionally, exposure to ophthalmology in undergraduate medical education continues to decrease and the increase in match rate could reflect the lack of proportional growth in the U.S. senior applicant pool compared with expansion of positions available. ${ }^{4,9}$ It is also possible that the increasing interest in other specialties could account for ophthalmology's increasing match rate. The creation of new specialties with a combination of relatively high income and controllable lifestyle, such as emergency medicine, or the increasing rate of compensation for other specialties, such as anesthesia, could account for the shift in medical student's specialty preference. ${ }^{10}$ Additionally, the implementation of the requirement of residency duty hours in 2003 could also have improved the attractiveness of traditionally less-desirable residencies. ${ }^{11}$

Previous work by Andriole et al addressed a similar question about surgical specialty preference by analyzing match rate trends from 1996 to 2000, but our results contrast with their findings. ${ }^{12}$ This study found an increasing match rate for ENT and a decreasing match rate for ophthalmology. It is difficult to make direct comparisons between our study and the study of Andriole et al due to different methodology and match rate definition as well as differing time periods studied. Our study evaluated match rate trends from 2009 to 2013 and the study of Andriole et al evaluated an earlier time period (1996-2000), which is notably prior to the implementation of residency duty hour requirements. Additionally, our study differs in the definition of match rate: the study of Andriole 
Table 1 Match data for graduating U.S. medical students, ophthalmology, and ENT from 2009 to 2013

\begin{tabular}{|l|l|l|l|l|l|}
\hline & Year & \multicolumn{1}{|l|}{} \\
\hline & 2009 & 2010 & 2011 & 2012 & 2013 \\
\hline Total U.S. medical student graduates & 16,168 & 16,467 & 16,836 & 17,363 & 17,341 \\
\hline Ophthalmology & \multicolumn{5}{|l|}{} \\
\hline Total spots & 459 & 458 & 461 & 461 & 460 \\
\hline Total U.S. senior applicants & 548 & 523 & 524 & 502 & 478 \\
\hline Total matched & $419(76.5 \%)$ & $413(79.0 \%)$ & $405(77.3 \%)$ & $411(81.9 \%)$ & $402(84.1 \%)$ \\
\hline ENT & \multicolumn{5}{|l|}{} \\
\hline Total spots & 275 & 280 & 283 & 285 & 292 \\
\hline Total U.S. senior applicants & 405 & 392 & 360 & 413 & 484 \\
\hline Total U.S. senior matched & $263(64.9 \%)$ & $259(66.1 \%)$ & $269(74.7 \%)$ & $277(67.1 \%)$ & $276(57.0 \%)$ \\
\hline
\end{tabular}

et al defined match rate as the quotient of students who matched over the number who ranked a program. Our study defined the match rate as those who matched over those who registered for the match process. We believe that our definition of match rate more accurately represents the true match rate because there are numerous students who desire to match in a certain specialty, register for the match in that specialty, receive no invitations to interview, and thus cannot rank a program. The study of Andriole et al did not include this proportion of students, which perhaps shifted the trend in the opposite direction of our trend.

Given that ophthalmology has traditionally been regarded as one of the most competitive specialties, the increasing odds of matching is surprising. ${ }^{10}$ Multiple studies have found that ophthalmology applicants are more likely to match if they have achieved criteria that make them a more competitive applicant such as Alpha-Omega-Alpha (AOA) membership, higher United States Medical Licensing Examination (USMLE) score, and attendance at top-ranked medical school. ${ }^{13,14}$ Although there has been an increase in the number of residency spots available, there has been an even greater increase in the number of graduating medical students. From 2009 to 2013, there was a less than $1 \%$ increase in ophthalmology residency spots and a $6 \%$ increase in ENT residency spots. However, there was an $11.7 \%$ increase in the number of graduating medical students, which suggests that the increase in ophthalmology match rate is unlikely to be a result of a larger number of residency spots. However, despite the increasing match rate, USMLE scores among ophthalmology applicants are increasing over time. This indicates that these data could represent a self-selecting process where some medical students may simply choose not to apply into a traditionally competitive field such as ophthalmology.

There are several limitations to this study. The primary limitation was the paucity of available match data. As we mentioned earlier, previous studies have defined match rate as the number of applicants matched divided by the number of applicants who submitted a rank list. These data are readily available on the National Residency Matching Program (NRMP) and SF Match web sites. However, we believe that this definition of match rate spuriously increases the calculated match rate because it misses the proportion of applicants who apply but receive no invitations to interview and thus cannot submit a rank list. An additional limitation of the study is the small number of surgical subspecialties included in this study. However, ENT and ophthalmology were the only surgical subspecialties in which the total number of applicants who registered for the match was available.

\section{Conclusion}

Overall, this study found that the odds of matching into the traditionally competitive field of ophthalmology are increasing among U.S. medical seniors, while odds of matching into the ENT have been decreasing. Identifying the factors behind these trends can have implications not only for these two surgical specialties but also for any specialty trying to increase its popularity among U.S. medical graduates.

\section{Funding}

This study was supported by an unrestricted grant from the Research to Prevent Blindness, New York, NY. Allison R. Loh is supported by unrestricted departmental funding from the Research to Prevent Blindness (New York, NY) and by grant P30 EY010572 from the National Institutes of Health (Bethesda, MD).

\section{Conflict of Interest}

None declared.

\section{Acknowledgments}

The authors thank the San Francisco Match Program (SF Match) and the Electronic Residency Application Service (ERAS) for their assistance with data collection.

\section{References}

1 Newton DA, Grayson MS. Trends in career choice by US medical school graduates. JAMA 2003;290(9):1179-1182 
2 Dorsey ER, Jarjoura D, Rutecki GW. Influence of controllable lifestyle on recent trends in specialty choice by US medical students. JAMA 2003;290(9):1173-1178

3 Schwartz RW, Haley JV, Williams C, et al. The controllable lifestyle factor and students' attitudes about specialty selection. Acad Med 1990;65(3):207-210

4 Shah M, Knoch D, Waxman E. The state of ophthalmology medical student education in the United States and Canada, 2012 through 2013. Ophthalmology 2014;121(6):1160-1163

5 The San Francisco Matching Program. Ophthalmology Residency Match Report-January 2009-2013. Available at: http://www. sfmatch.org/residency/ophthalmology/about_match/match_report.pdf. Accessed March 4, 2013

6 Results and Data: Main Residency Match. NRMP Historical Reports 2009-2013. Available at: http://www.nrmp.org/data/historicalreports.html. Accessed March 4, 2013

7 FACTS: Applicants, Matriculants, Enrollment, Graduates, M.D.-Ph.D., and Residency Applicants Data. Available at: https://www.aamc.org/ data/facts/. Accessed March 4, 2013
8 Ebell MH. Future salary and US residency fill rate revisited. JAMA 2008;300(10):1131-1132

9 Mottow-Lippa L. Ophthalmology in the medical school curriculum: reestablishing our value and effecting change. Ophthalmology 2009;116(7):1235-1236, 1236.e1

10 Patel MS, Katz JT, Volpp KG. Match rates into higher-income, controllable lifestyle specialties for students from highly ranked, research-based medical schools compared with other applicants. J Grad Med Educ 2010;2(3):360-365

11 Fabricant PD, Dy CJ, Dare DM, Bostrom MP. A narrative review of surgical resident duty hour limits: where do we go from here? J Grad Med Educ 2013;5(1):19-24

12 Andriole DA, Schechtman KB, Ryan K, Whelan A, Diemer K. How competitive is my surgical specialty? Am J Surg 2002;184(1):1-5

13 Loh AR, Joseph D, Keenan JD, Lietman TM, Naseri A. Predictors of matching in an ophthalmology residency program. Ophthalmology 2013;120(4):865-870

14 Yousuf SJ, Jones LS. Ophthalmology Residency Match outcomes for 2011. Ophthalmology 2012;119(3):642-646 